\title{
The flat closing problem for buildings
}

\author{
CORINA CIOBOTARU
}

\begin{abstract}
Using the notion of a strongly regular hyperbolic automorphism of a locally finite Euclidean building, we prove that any (not necessarily discrete) closed, cocompact subgroup of the type-preserving automorphisms group of a locally finite general nonspherical building contains a compact-by- $\mathbf{Z}^{d}$ subgroup, where $d$ is the dimension of a maximal flat.
\end{abstract}

57S25, 51E24; 20E42, 20F55

\section{Introduction}

In 1972, Prasad and Raghunathan [7] proved the following result (see [7, Corollary 2.9, Lemma 1.15]):

Let $G$ be a semisimple real Lie group of rank $r$ (which may admit compact factors). Let $\Gamma<G$ be a lattice. Then $\Gamma$ contains an abelian subgroup of rank $r$.

To obtain this result, they use in the first place the existence in $\Gamma$ of a so-called $\mathbf{R}$-hyperregular element (see Prasad and Raghunathan [7, Definition 1.1 and Theorem 2.5]). Being also an $\mathbf{R}$-regular element (see [7, Remark 1.2] and Steinberg [10]), an $\mathbf{R}$-hyperregular $g \in G$ inherits the property that its $G$-centralizer $\operatorname{Cent}_{G}(g)$ contains a unique maximal $\mathbf{R}$-split torus of $G$ of dimension equal to the $\mathbf{R}$-rank of $G$ (see [10] or [7]). Using this, the last step of Prasad and Raghunathan's strategy is to show that $\operatorname{Cent}_{G}(g) / \operatorname{Cent}_{\Gamma}(g)$ is compact if $g$ is an $\mathbf{R}$-hyperregular element (see [7, proof of Lemma 1.15]). This is obtained in the following way. If $\Gamma$ is a uniform lattice then one can use Selberg's lemma (see [7, Lemma 1.10]). If not, then [7, Theorem 1.14] gives the desired result.

The above result of Prasad and Raghunathan can be related, in the setting of CAT(0) spaces, to the following question of Gromov [5, Section 6. $\mathrm{B}_{3}$ ] (also known as the flat closing problem). From another point of view, the flat closing problem is a converse to the flat torus theorem.

First, let us recall the following basic notions from the setting of CAT(0) spaces. 
1.1 Definition Let $X$ be a proper CAT(0) space and $G$ be a not necessarily discrete locally compact group acting continuously and properly by isometries on $X$. By a geometric flat $F \subset X$ of dimension $d$ (or a $d$-flat) we mean a closed convex subset of $X$ which is isometric to the Euclidean $d$-space, where $d \geq 2$. Moreover, we say that a subset $Y \subset X$ is periodic if $\operatorname{Stab}_{G}(Y)$ acts cocompactly on $Y$.

When $\gamma$ is a hyperbolic isometry of $X$ we define

$$
\operatorname{Min}(\gamma):=\left\{x \in X\left|\operatorname{dist}_{X}(x, \gamma(x))=\right| \gamma \mid\right\},
$$

where $|\gamma|:=\inf _{x \in X} \operatorname{dist}_{X}(x, \gamma(x))$ denotes the translation length of $\gamma$.

1.2 Problem (The flat closing problem) Let $X$ be a proper CAT(0) space and $\Gamma$ be a discrete group acting continuously, properly and cocompactly by isometries on $X$. Does the existence of a $d$-flat $F \subset X$ imply that $\Gamma$ contains a copy of $\mathbf{Z}^{d}$ ?

Remark In the hypotheses of Problem 1.2, note that if $F$ is a periodic $d$-flat then indeed, by the Bieberbach theorem, $\operatorname{Stab}_{\Gamma}(F)$ contains a copy of $\mathbf{Z}^{d}$ and therefore $\Gamma$ contains a subgroup that is virtually $\mathbf{Z}^{d}$. Moreover, if we replace $\Gamma$ with a not necessarily discrete locally compact group $G$ acting continuously, properly and cocompactly by isometries on $X$ and $F$ is a periodic $d$-flat with respect to the $G$-action, then $G$ contains a compact-by- $\mathbf{Z}^{d}$ subgroup.

To attack Gromov's Problem 1.2, the natural strategy would be to construct periodic flats or more generally periodic subspaces of the form $Y=F \times C \subset X$, where $F$ is a $d$-flat and $C$ is a compact set. This could be done by showing that $\Gamma$ contains a hyperbolic element $\gamma$ which is "regular" (see Definition 2.1) and then considering $Y:=\operatorname{Min}(\gamma)=F \times C$, where $F$ is a flat and $C$ is a compact set. To conclude from here that the flat closing problem has an affirmative answer it would be enough to show that the centralizer of $\gamma$ in $\Gamma$, namely $\operatorname{Cent}_{\Gamma}(\gamma)<\operatorname{Stab}_{\Gamma}(Y)$, acts cocompactly on $Y$ and that $\operatorname{Stab}_{\operatorname{Cent}_{\Gamma}(\gamma)}(F) \leq \operatorname{Stab}_{\Gamma}(F)$ has cocompact action on $F$. Thus, $\operatorname{Stab}_{\Gamma}(F)$ would contain a copy of $\mathbf{Z}^{d}$ and the remark applies.

Notice that the above strategy is analogous to the one used in the Prasad-Raghunathan result, where the $\mathbf{R}$-split torus is replaced with a flat. Moreover, this strategy, including the existence of "regular" elements, is successfully implemented for example in Caprace and Zadnik [4], where it is proved that any discrete group acting properly and cocompactly on a decomposable locally compact CAT(0) space which admits in addition the geodesic extension property (ie every geodesic segment is contained in a bi-infinite geodesic line) contains virtually $\mathbf{Z}^{d}$, where $d$ is the number of indecomposable de Rham factors. Notice that in general the dimension of a maximal flat of a CAT(0) space is bigger than the number of the indecomposable de Rham factors. 
In this article we propose to answer further Gromov's Problem 1.2 in the case when $G$ is a not necessarily discrete locally compact group acting continuously, properly and cocompactly by type-preserving automorphisms on a locally finite general nonspherical building. We stress here that we cannot apply directly the result of Caprace and Zadnik [4]. Firstly, because in the usual Davis realization, buildings are CAT(0) spaces where in general the geodesic extension property is not fulfilled. Secondly, the group $G$ considered in this article is not necessarily discrete. Thirdly, we exploit two other ingredients that are missing in the context of Caprace and Zadnik [4]: the case of Euclidean buildings and the reduction to products in the nonaffine part. Still, the strategy is the same and uses the existence of "strongly regular hyperbolic automorphisms" (see Definition 2.2 below) acting on locally finite Euclidean buildings. We obtain the following theorem:

1.3 Theorem (See Theorem 3.4) Let $\Delta$ be a locally finite building of nonspherical type $(W, S)$, but with $S$ being finite. Let $G$ be a closed, not necessarily discrete, type-preserving subgroup of $\operatorname{Aut}(\Delta)$, with cocompact action on $\Delta$. Then $G$ contains a compact-by- $\mathbf{Z}^{d}$ subgroup, where $d$ is the dimension of a maximal flat of $\Delta$.

\section{Preliminaries}

We start by recalling briefly some basic definitions and results from Caprace and Ciobotaru [2] on strongly regular hyperbolic automorphisms acting on locally finite Euclidean buildings, as they are used further in this paper. For some notation see also Definition 1.1 from Section 1.

2.1 Definition Let $X$ be a CAT(0) space and $\gamma$ be a hyperbolic isometry of $X$. We say that $\gamma$ is a regular hyperbolic isometry if $\operatorname{Min}(\gamma)$ is a bounded Hausdorff distance from a maximal flat of $X$.

In the particular case of locally finite Euclidean buildings we have the following stronger definition introduced in Caprace and Ciobotaru [2].

2.2 Definition Let $\Delta$ be a Euclidean building and $\gamma \in \operatorname{Aut}(\Delta)$ be a type-preserving automorphism. We say that $\gamma$ is a strongly regular hyperbolic automorphism if $\gamma$ is a hyperbolic isometry and the two endpoints of one (and hence all) of its translation axes lie in the interior of two opposite chambers of the spherical building at infinity. In $\operatorname{particular} \operatorname{Min}(\gamma)$ is an apartment of $\Delta$ and is uniquely determined.

In addition, if $\ell$ is a geodesic line of the Euclidean building $\Delta$, we say that $\ell$ is strongly regular if its endpoints lie in the interior of two opposite chambers of the spherical building at infinity of $\Delta$. 
2.3 Definition Let $X$ be a $\operatorname{CAT}(0)$ space. A geodesic line $\ell$ in $X$ is said to have rank one if it does not bound a flat half-plane. An isometry $\gamma \in \operatorname{Is}(X)$ is said to have rank one if it is hyperbolic and if some (and hence any) of its axes has rank one.

The next two results from Caprace and Ciobotaru [2] will be used in what follows.

2.4 Lemma (See [2, Lemma 2.6]) Let $(W, S)$ be a Euclidean Coxeter system. Then $W$ contains strongly regular hyperbolic elements.

2.5 Proposition (See [2, Proposition 2.9]) Let $X$ be a proper CAT(0) space, $G \leq \operatorname{Is}(X)$ be any subgroup of isometries and $\rho: \mathbf{R} \rightarrow X$ be a geodesic map. Assume there is an increasing sequence $\left\{t_{n}\right\}_{n \geq 0}$ of positive real numbers tending to infinity such that $\sup _{n} d\left(\rho\left(t_{n}\right), \rho\left(t_{n+1}\right)\right)<\infty$ and that the set $\left\{\rho\left(t_{n}\right)\right\}_{n \geq 0}$ falls into finitely many $G$-orbits, each of which is moreover discrete.

Then there is an increasing sequence $\{f(n)\}_{n}$ of positive integers such that, for all $n>m>0$, there is a hyperbolic isometry $h_{m, n} \in G$ which has a translation axis containing the geodesic segment $\left[\rho\left(t_{f(m)}\right), \rho\left(t_{f(n)}\right)\right]$.

In addition, if the geodesic line $\rho(\mathbf{R})$ is the translation axis of a rank-one element of Is $(X)$, then for each fixed $m>0$ there exists $N_{m}>0$ such that for every $n>N_{m}$ the isometry $h_{m, n}$ is a rank-one element. Moreover, if $X$ is a locally finite Euclidean building and the geodesic line $\rho(\mathbf{R})$ is strongly regular, then $h_{m, n}$ is a strongly regular hyperbolic automorphism.

Proof We give a proof only for the very last assertion of the proposition, this not being part of [2, Proposition 2.9].

Let $m$ be fixed. Suppose the contrary, namely for every $k>0$ there exists $n_{k} \geq k$ such that $h_{m, n_{k}}$ is not a rank-one element. This means that the translation axis of $h_{m, n_{k}}$ containing the geodesic segment $\left[\rho\left(t_{f(m)}\right), \rho\left(t_{f\left(n_{k}\right)}\right)\right]$ is contained in the boundary of a flat half-plane. Therefore, as $k \rightarrow \infty$ we obtain that the geodesic ray $\left[\rho\left(t_{f(m)}\right), \rho(\infty)\right)$ is contained in the boundary of a flat half-plane as well. As the space $X$ is proper and the geodesic line $\rho(\mathbf{R})$ is the translation axis of a rank-one hyperbolic element, we obtain a contradiction with the fact that the diameter of the projection on $\rho(\mathbf{R})$ of every closed metric ball in $X$ which is moreover disjoint from $\rho(\mathbf{R})$ must be bounded above by a fixed constant. The conclusion follows. 


\section{The proof of the main theorem}

Before proceeding to the proof of Theorem 1.3, we recall some general facts about buildings and we fix some notation. Let $\Delta$ be a locally finite general building of type $(W, S)$, with $S$ finite. Fix from now on a chamber $\mathbf{c}$ in $\operatorname{Ch}(\Delta)$. Let $W=W_{1} \times W_{2} \times \cdots \times W_{k}$ be the direct product decomposition of $W$ in irreducible Coxeter systems $\left(W_{i}, S_{i}\right)$. Thus $S=S_{1} \sqcup S_{2} \sqcup \cdots \sqcup S_{k}$ is a disjoint union. Denote by $\Delta_{i}$ the $W_{i}$-residue in $\Delta$ containing the fixed chamber c. From Ronan [8, Theorem 3.10] we have that $\Delta \cong \Delta_{1} \times \cdots \times \Delta_{k}$. For what follows, we use the notation

$$
\begin{aligned}
\mathbf{E u c l} & =\left\{i \in\{1, \ldots, k\} \mid\left(W_{i}, S_{i}\right) \text { is Euclidean }\right\}, \\
\mathbf{S p h} & =\left\{i \in\{1, \ldots, k\} \mid\left(W_{i}, S_{i}\right) \text { is finite }\right\}, \\
\mathbf{n S p h} & =\{1, \ldots, k\} \backslash \mathbf{S p h}, \\
\mathbf{n E s p h} & =\{1, \ldots, k\} \backslash(\text { Eucl } \cup \mathbf{S p h}) .
\end{aligned}
$$

Accordingly, we use the notation $\Delta_{\mathbf{A}}:=\prod_{i \in \mathbf{A}} \Delta_{i}$ and $W_{\mathbf{A}}:=\prod_{i \in \mathbf{A}} W_{i}$, where $\mathbf{A}$ is one of the sets Eucl, Sph, $\mathbf{n S p h}$ or nEsph. Moreover, for every $i \in\{1, \ldots, k\}$ we denote by $\operatorname{pr}_{i}: \Delta \rightarrow \Delta_{i}$ the projection map on $\Delta_{i}$ and by abuse of notation we write $\operatorname{pr}_{i}(\gamma)$ to represent a type-preserving element $\gamma \in \operatorname{Aut}(\Delta)$ acting on the factor $\Delta_{i}$.

The first step towards the main theorem is given by the next proposition, which uses an argument of Hruska and Kleiner [6, Lemma 3.1.2].

3.1 Proposition Let $\Delta$ be a locally finite building of nonspherical type $(W, S)$, but with $S$ being finite. Let $G$ be a not necessarily discrete, type-preserving subgroup of $\operatorname{Aut}(\Delta)$ acting cocompactly on $\Delta$ and let $\mathcal{R}$ be any residue in $\Delta$ containing the chamber c. Then $\operatorname{Stab}_{G}(\mathcal{R})$ acts cocompactly on $\mathcal{R}$.

Proof Let us denote by $\left(W^{\prime}, S^{\prime}\right)$ the type of the residue $\mathcal{R}$, where $W^{\prime} \leq W$ and $S^{\prime} \subset S$. Take $K \subset \Delta$ to be a compact fundamental domain corresponding to the action of $G$ and containing the chamber c. Let $\left(g_{i}\right)_{i \in I}$ be a subset of $G$ such that $\mathcal{R} \subset \bigcup_{i \in I} g_{i}(K)$. Because $\mathcal{R}$ is a residue containing $\mathbf{c}$ and $G$ is type-preserving, for every $i \in I, g_{i}^{-1}(\mathcal{R})$ is a residue of the same type as $\mathcal{R}$, containing the chamber $g_{i}^{-1}(\mathbf{c})$ and intersecting $K$.

Notice that a compact set $K$ of a (not necessarily locally finite) building always has a finite number of chambers. Therefore, $K$ intersects a finite number of $\left(W^{\prime}, S^{\prime}\right)$-type residues of $\Delta$. We conclude that there is a finite number of left cosets of the form $\operatorname{Stab}_{G}(\mathcal{R}) g_{i}$, with $i \in I$. Denote by $\left\{g_{1}, \ldots, g_{t}\right\} \subset\left\{g_{i}\right\}_{i \in I}$ the finite set of representatives of these left cosets. We obtain that $\mathcal{R}$ is covered by $\bigcup_{j \in\{1, \ldots, t\}} \operatorname{Stab}_{G}(\mathcal{R}) g_{j}(K)$. 
Because $g_{j}(K)$ is compact, let $K^{\prime}$ be a compact in $\Delta$ such that $\bigcup_{j=1}^{t} g_{j}(K) \subset K^{\prime}$. Thus $\operatorname{Stab}_{G}(\mathcal{R}) K^{\prime}$ covers $\mathcal{R}$ and the conclusion follows.

The second step is to find a hyperbolic element in $\operatorname{Stab}_{G}\left(\Delta_{\mathbf{n S p h}}\right)$. This is given by the following proposition.

3.2 Proposition Let $\Delta$ be a locally finite building of nonspherical type $(W, S)$, but with $S$ being finite. Let $G$ be a not necessarily discrete, type-preserving subgroup of $\operatorname{Aut}(\Delta)$ acting cocompactly on $\Delta$. Then there exists a hyperbolic element $\gamma \in \operatorname{Stab}_{G}\left(\Delta_{\mathbf{n S p h}}\right)$ such that $\operatorname{pr}_{i}(\gamma)$ is a strongly regular hyperbolic element if $i \in$ Eucl and a rank-one isometry if $i \in \mathbf{n E s p h}$.

Proof First, notice that $G$ acts cocompactly on $\Delta_{\mathbf{n S p h}}$. Take $i \in$ Eucl. By Lemma 2.4, let $l_{i}$ be a strongly regular geodesic line contained in some apartment of $\Delta_{i}$, constructed using a strongly regular hyperbolic element $\gamma_{i}$ of $W_{i}$. The line $l_{i}$ is thus contained in a unique apartment of $\Delta_{i}$. Denote by $\left\{v_{i, j}\right\}_{j \in \mathbf{Z}}$ a bi-infinite sequence of points in $1_{i}$ such that $\gamma_{i}\left(v_{i, j}\right)=v_{i, j+1}$, for every $j \in \mathbf{Z}$. For example, the points $\left\{v_{i, j}\right\}_{j \in \mathbf{Z}} \subset 1_{i}$ can be taken to be special vertices of $\Delta_{i}$, of the same type.

For $i \in \mathbf{n E s p h}$ we have a similar construction. Following Caprace and Fujiwara [3, Proposition 4.5], denote by $r_{i}$ a rank-one geodesic line given by a rank-one hyperbolic element $h_{i} \in W_{i}$. Denote by $\left\{t_{i, j}\right\}_{j \in \mathbf{Z}}$ a bi-infinite sequence of points of $r_{i}$ such that $h_{i}\left(t_{i, j}\right)=t_{i, j+1}$, for every $j \in \mathbf{Z}$.

Because $\prod_{i \in \text { Eucl }} l_{i} \times \prod_{i \in \mathbf{n E s p h}} r_{i}$ is a flat of $\Delta_{\mathbf{n S p h}}$, we consider in its interior the infinite geodesic line determined by the sequence of points

$$
\left\{o_{j}:=\prod_{i \in \text { Eucl }} v_{i, j} \times \prod_{i \in \mathbf{n E s p h}} t_{i, j}\right\}_{j \in \mathbf{Z}} \subset \Delta_{\mathbf{n S p h}} .
$$

Denote the resulting geodesic line by L and observe that, by defining

$$
h:=\prod_{i \in \text { Eucl }} \gamma_{i} \times \prod_{i \in \mathbf{n E s p h}} \gamma_{i}
$$

we have that $h \in W_{\mathbf{n S p h}}$ and $h\left(o_{j}\right)=o_{j+1}$, for every $j \in \mathbf{Z}$.

We are now ready to proceed in finding the desired hyperbolic element in $G$. Apply Proposition 2.5 to our geodesic line L, to the sequence of points $\left\{o_{j}\right\}_{j \in \mathbf{N}}$ and to the $\operatorname{Stab}_{G}\left(\Delta_{\mathbf{n S p h}}\right)$-action on $\Delta_{\mathbf{n S p h}}$ (which is cocompact). As we are working with a locally finite building, all hypotheses of Proposition 2.5 are fulfilled. We obtain thus a sequence $\{f(n)\}_{n \geq 0}$ and a sequence of hyperbolic elements $\left\{\gamma_{m, n}\right\}_{0<m<n} \subset \operatorname{Stab}_{G}\left(\Delta_{\mathbf{n S p h}}\right)$ such that every $\gamma_{m, n}$ has a translation axis containing the geodesic segment $\left[o_{f(m)}, o_{f(n)}\right]$. 
By this construction we obtain that $\operatorname{pr}_{i}\left(\gamma_{m, n}\left(o_{f(m)}\right)\right)=\operatorname{pr}_{i}\left(o_{f(n)}\right)$, for every $i \in \mathbf{n S p h}$ and every element $\gamma_{m, n}$. Applying again Proposition 2.5, there exists a hyperbolic element $\gamma_{m, n}$ such that $\operatorname{pr}_{i}\left(\gamma_{m, n}\right)$ is a strongly regular hyperbolic element if $i \in$ Eucl and a rank-one isometry if $i \in \mathbf{n E s p h}$. The conclusion follows.

Before starting the proof of the main Theorem 3.4, let us mention that the power $d$ of the "compact-by- $\mathbf{Z}^{d}$ subgroup" appearing in the conclusion of the theorem is maximal with respect to the $d$-flats of the building $\Delta$. The maximality is explained by the following result.

3.3 Proposition (See Caprace [1, Proposition 3.1]) Let $F$ be a maximal $d$-flat of a locally finite general building $\Delta$. Then there exists a residue $\mathcal{R} \subset \Delta$ of type $\left(W_{\mathcal{R}}, S_{\mathcal{R}}\right)$ such that $d=\sum_{i \in \mathbf{E u c l}_{\mathcal{R}}} n_{i}+\left|\mathbf{n} \mathbf{E s p h}_{\mathcal{R}}\right|$, where $\mathbf{E u c l}_{\mathcal{R}}$ and $\mathbf{n} \mathbf{E s p h}_{\mathcal{R}}$ correspond to the residue $\mathcal{R}$.

Therefore, by Propositions 3.1 and 3.3, to answer Gromov's flat closing problem in the case of a locally finite general nonspherical building it is enough to prove the following theorem.

3.4 Theorem Let $\Delta$ be a locally finite building of nonspherical type $(W, S)$, with $S$ being finite, and $G$ a closed, not necessarily discrete, type-preserving subgroup of $\operatorname{Aut}(\Delta)$ with cocompact action. Then $G$ contains a compact-by- $\mathbf{Z}^{d}$ subgroup, where $d:=\sum_{i \in \mathbf{E u c l}} n_{i}+|\mathbf{n E s p h}|$.

Proof Let $\gamma \in \operatorname{Stab}_{G}\left(\Delta_{\mathbf{n S p h}}\right)$ be a hyperbolic element given by Proposition 3.2. We have that $\operatorname{Min}(\gamma)=\prod_{i \in \text { Eucl }} \mathbf{R}^{n_{i}} \times \prod_{j \in \mathbf{n E s p h}}\left(\mathbf{R} \times C_{j}\right)$, where $n_{i}$ is the Euclidean dimension of the corresponding building $\Delta_{i}$ and $C_{j}$ is a compact, convex subset of the corresponding building $\Delta_{j}$. Let $F:=\prod_{i \in \text { Eucl }} \mathbf{R}^{n_{i}} \times \prod_{i \in \mathbf{n E s p h}} \mathbf{R} \subset \operatorname{Min}(\gamma)$. By Ruane [9, Theorem 3.2], whose proof works also for not necessarily discrete groups, we have that $\operatorname{Cent}_{G}(\gamma)$ stabilizes and acts cocompactly on $\operatorname{Min}(\gamma)$. As $\prod_{i \in \mathbf{n E s p h}} C_{i}$ is compact, using the argument of Hruska and Kleiner [6, Lemma 3.1.2], recalled in the proof of Proposition 3.1, and the fact that we are working with CAT(0) cellular complexes we obtain that $\operatorname{Stab}_{\mathrm{Cent}_{G}(\gamma)}(F)$ acts cocompactly on $F$. In particular, we obtain that the action of $\operatorname{Stab}_{G}(F)$ on $F$ is properly discontinuous and cocompact. Therefore, the group $\operatorname{Stab}_{G}(F) / \operatorname{Fix}_{G}(F)$ is virtually isomorphic with $\mathbf{Z}^{d}$, where $d:=\sum_{i \in \text { Eucl }} n_{i}+|\mathbf{n E s p h}|$. Since $G$ is closed, the $\operatorname{group}_{\operatorname{Fix}_{G}}(F)$ is compact. Therefore, the group $G$ contains a compact-by- $\mathbf{Z}^{d}$ subgroup. This concludes the proof of the theorem. 
Acknowledgements We would like to thank Pierre-Emmanuel Caprace for proposing this question, for his comments and useful discussions, Gašper Zadnik for some useful explanations about CAT(0) spaces and the referee for his/her wise comments. The author is supported by the FRIA.

\section{References}

[1] P-E Caprace, Buildings with isolated subspaces and relatively hyperbolic Coxeter groups, Innov. Incidence Geom. 10 (2009) 15-31 MR2665193

[2] P-E Caprace, C Ciobotaru, Gelfand pairs and strong transitivity for euclidean buildings arXiv:1304.6210 To appear in "Ergodic Theory and Dynamical Systems"

[3] P-E Caprace, K Fujiwara, Rank-one isometries of buildings and quasi-morphisms of Kac-Moody groups, Geom. Funct. Anal. 19 (2010) 1296-1319 MR2585575

[4] P-E Caprace, G Zadnik, Regular elements in CAT(0) groups, Groups Geom. Dyn. 7 (2013) 535-541 MR3095707

[5] M Gromov, Asymptotic invariants of infinite groups, from: "Geometric group theory, Vol. 2", (G A Niblo, M A Roller, editors), London Math. Soc. Lecture Note Ser. 182, Cambridge Univ. Press (1993) 1-295 MR1253544

[6] G C Hruska, B Kleiner, Hadamard spaces with isolated flats, Geom. Topol. 9 (2005) 1501-1538 MR2175151

[7] G Prasad, MS Raghunathan, Cartan subgroups and lattices in semi-simple groups, Ann. of Math. 96 (1972) 296-317 MR0302822

[8] M Ronan, Lectures on buildings, Perspectives in Mathematics 7, Academic Press, Boston (1989) MR1005533

[9] KE Ruane, Dynamics of the action of a CAT(0) group on the boundary, Geom. Dedicata 84 (2001) 81-99 MR1825346

[10] R Steinberg, Regular elements of semisimple algebraic groups, Inst. Hautes Études Sci. Publ. Math. (1965) 49-80 MR0180554

Institut de recherche en mathématique et physique (IRMP), UCL

Chemin du Cyclotron 2, bte L7.01.01, 1348 Louvain-la-Neuve, Belgium corina.ciobotaru@gmail.com

Received: 5 November 2013 Revised: 20 March 2014 